\title{
Assessment of Feasibility of Endovascular Treatment of Ruptured Intracranial Aneurysms with 16-Detector Row CT Angiography
}

\author{
Mathieu van der Jagt ${ }^{a, d} \quad H$. Zwenneke Flach $^{b} \quad$ Hervé L.J. Tanghe ${ }^{b}$

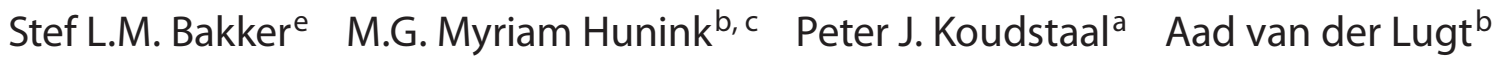 \\ Departments of a Neurology, ${ }^{\mathrm{b}}$ Radiology, ${ }^{\mathrm{C}}$ Epidemiology and Biostatistics, and ${ }^{\mathrm{d}}$ Intensive Care, Erasmus MC, and \\ e Department of Neurology, Sint Franciscus General Hospital, Rotterdam, The Netherlands
}

\section{Key Words}

Endovascular treatment • Intracranial aneurysm •

CT angiography $\cdot$ Subarachnoid hemorrhage

\begin{abstract}
Background: It is unclear whether 16-detector row CT angiography (CTA) can replace digital subtraction angiography (DSA) to assess the feasibility of endovascular treatment (EVT) in the acute phase after aneurysmal subarachnoid hemorrhage. Methods: We studied 80 consecutive patients with aneurysmal subarachnoid hemorrhage, who underwent both CTA and DSA. Two interventional neuroradiologists independently scored CTA and, immediately thereafter, DSA with respect to feasibility of EVT. We determined whether CTA without DSA was sufficient for a definite judgment. We also assessed interobserver agreement. Results: The 2 readers judged EVT to be feasible in 24 and 37 patients with CTA alone and not feasible in 34 and 20 patients. In these patients, DSA yielded additional information in 6 (reader 1 ) and 5 patients (reader 2), which did not affect treatment decision. In 19 and 7 patients, DSA was considered inferior to CTA. In the remaining patients $(n=22$ and 23, respectively), feasibility of EVT could not be judged with CTA
\end{abstract}

alone, and DSA results were required in addition for a treatment decision. Interobserver agreement on feasibility of EVT was just fair $(\kappa<0.40)$. Conclusions: In our series of patients, 16-detector row CTA was a reliable investigation to assess feasibility of EVT of ruptured intracranial aneurysms in most patients. Further, we found that interobserver disagreement on feasibility of EVT was considerable.

Copyright $\odot 2008$ S. Karger AG, Basel

\section{Introduction}

In patients with aneurysmal subarachnoid hemorrhage (SAH), CT angiography (CTA) confers substantial advantage over digital subtraction angiography (DSA) because it is quick, safe and diminishes demands on conventional angiographic resources [1]. The advent of multidetector row CT scanners has improved the diagnostic accuracy of CT $[1,2]$. Equivalent diagnostic accuracy for the detection of intracranial aneurysms has been reported with 4-and 16-detector row CTA compared with DSA [3-6]. However, the diagnostic value of CTA also depends on the ability to select the appropriate treatment [1]. The recent shift from surgical to endovascular treatment

\section{KARGER}

Fax +4161306 1234 E-Mail karger@karger.ch www.karger.com

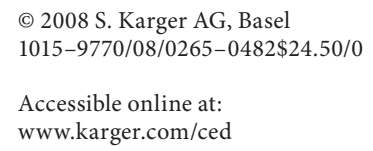

M. van der Jagt

Department of Intensive Care

Erasmus MC University Medical Center

s-Gravendijkwal 230, NL-3015 CE Rotterdam (The Netherlands)

Tel. +31 10704 0704, Fax +31 10463 2156, E-Mail m.vanderjagt@erasmusmc.nl 
(EVT) as first-line treatment [7] may have altered the requirements posed on angiographic visualization of intracranial aneurysm. The diagnostic value of the more recently developed high-resolution multidetector row CT scanners with respect to the clinical assessment regarding the feasibility of EVT of the ruptured aneurysm has not been extensively studied. Therefore, we investigated the value of 16-detector row CTA for the assessment of feasibility of EVT of ruptured intracranial aneurysms.

\section{Materials and Methods}

\section{Patient Inclusion}

We performed a prospective observational cohort study. Patients were recruited from 1 October 2002 to 10 September 2004 in a University Hospital. Since 2002, it is standard clinical practice to perform both CTA and DSA in all patients with SAH of presumed aneurysmal origin, provided that their clinical condition permits the performance of both procedures. EVT was considered the preferred treatment option for ruptured intracranial aneurysms [7]. Inclusion criteria for this study were: (1) clinical diagnosis of $\mathrm{SAH}$, confirmed by CT or cerebrospinal fluid spectrophotometric analysis; (2) age $\geq 18$ years; (3) written informed consent by the patient or relative to review the patient's data; (4) performance of both CTA and DSA, and (5) an aneurysm confirmed with CTA and DSA. The protocol was approved by the medical ethics committee of the hospital.

\section{CTA Data Acquisition}

Studies were performed with a 16-detector row CT scanner (Somatom X-32 Sensation 16; Siemens Medical Solutions, Erlangen, Germany). The scan volume started from the upper limit of the posterior arch of the atlas and extended cephalad with a coverage of $100 \mathrm{~mm}$. The lower limit was chosen to include a proximal origin of the posterior inferior cerebellar artery; the upper limit was chosen to include the callosomarginal artery. Eighty milliliters of contrast material (Iodixanol $320 \mathrm{mg} / \mathrm{ml}$, Visipaque; Amersham Health, Little Chalfont, UK) was injected. The CTA scan was synchronized with the contrast material injection with a bolus tracking technique, with a $40-\mathrm{ml}$ saline bolus chaser $(4 \mathrm{ml} / \mathrm{s})$.

\section{DSA Data Acquisition}

Four-vessel DSA was done according to the Seldinger technique. A 4- to 5-french catheter was selectively placed in the internal carotid and the vertebral arteries, injecting $6 \mathrm{ml}(4 \mathrm{ml} / \mathrm{s}$; internal carotid) or $7 \mathrm{ml}(5 \mathrm{ml} / \mathrm{s}$; vertebral artery) of contrast material (Iomeron 350; Bracco, Milan). In all patients, frontal, lateral and oblique views were obtained (matrix $1,024 \times 1,024$; image intensifier $20-28 \mathrm{~cm}$ ).

\section{Data Scoring and Evaluation}

Two experienced interventional neuroradiologists independently scored the CTA images and, immediately thereafter, the DSA images. Both readers have been familiar with the clinical use of the CTA for more than 3 years. Reader 1 had performed over
100 endovascular procedures in patients with ruptured aneurysms and reader 2 more than 40 procedures. Both readers were unaware of the clinical condition of the patients and actual treatment but were aware of the results of the unenhanced diagnostic CT. CTA images were examined at a work station. A standardized evaluation was performed with maximum intensity projections with a thickness of $6-8 \mathrm{~mm}$ and an overlap of 3-4 $\mathrm{mm}$ in axial, sagittal and coronal planes for the anterior circulation, as well as in the sagittal plane and a plane parallel to the clivus for the posterior circulation. Maximum intensity projection planes and thickness could be adjusted and source images were available for additional evaluation.

DSA images were examined on hard copy films. Both the CTA images and the DSA hard copies were masked for patient identification.

\section{CT Angiography}

First, the readers scored the CTA on the work station blinded to the DSA. The CTA was evaluated with respect to the location and size of the target aneurysm, which was defined as the aneurysm that had ruptured according to the reader. The location of the target aneurysm was noted as being at 1 of the 29 locations, as previously described by Yasargil [8].

The size of the target aneurysm (length from fundus to base in millimeters) and the presence of vasospasm were measured by one of the neuroradiologists. Vasospasm was evaluated because this may influence both the judgment of suitability for EVT and the quality of CTA and DSA.

In general, criteria of feasibility of EVT included a discernible aneurysmal neck and the absence of vessels sprouting from the aneurysmal sac. Both readers chose 1 of the following 3 options with regard to suitability of the target aneurysm for EVT (fig. 1): (1) feasible (with or without balloon remodeling technique) and DSA not necessary, (2) not feasible and DSA not necessary, or (3) additional DSA needed. When option 2 or 3 was chosen, the reason for this choice was also specified.

\section{Objective}

The main objective was to assess the proportion of target aneurysms for which 16-detector row CTA was regarded as sufficient by each reader separately to make a definite judgment on feasibility of EVT. In addition, we assessed interobserver agreement with respect to feasibility judgments based on CTA only and on both CTA and DSA. Further, we assessed: (1) how often and for what reason examination of DSA changed a definite judgment on EVT feasibility with CTA; (2) additional information provided by DSA; (3) reasons for requiring DSA, and (4) the relation between the size and location of the target aneurysm and vasospasm and the proportion of definite judgments that could be made with CTA.

\section{Statistics}

Statistical analysis was performed with SPSS 12.0.1. Interobserver agreement between readers regarding EVT feasibility was assessed with Cohen's kappa (к) [9]. The proportion of patients in whom definite judgments regarding EVT feasibility could be made with CTA alone, and next after viewing DSA, were tabulated in $2 \times 2$ tables. The relationship between the readers' judgments and characteristics of the aneurysms were tested with Pearson's $\chi^{2}$ test. 


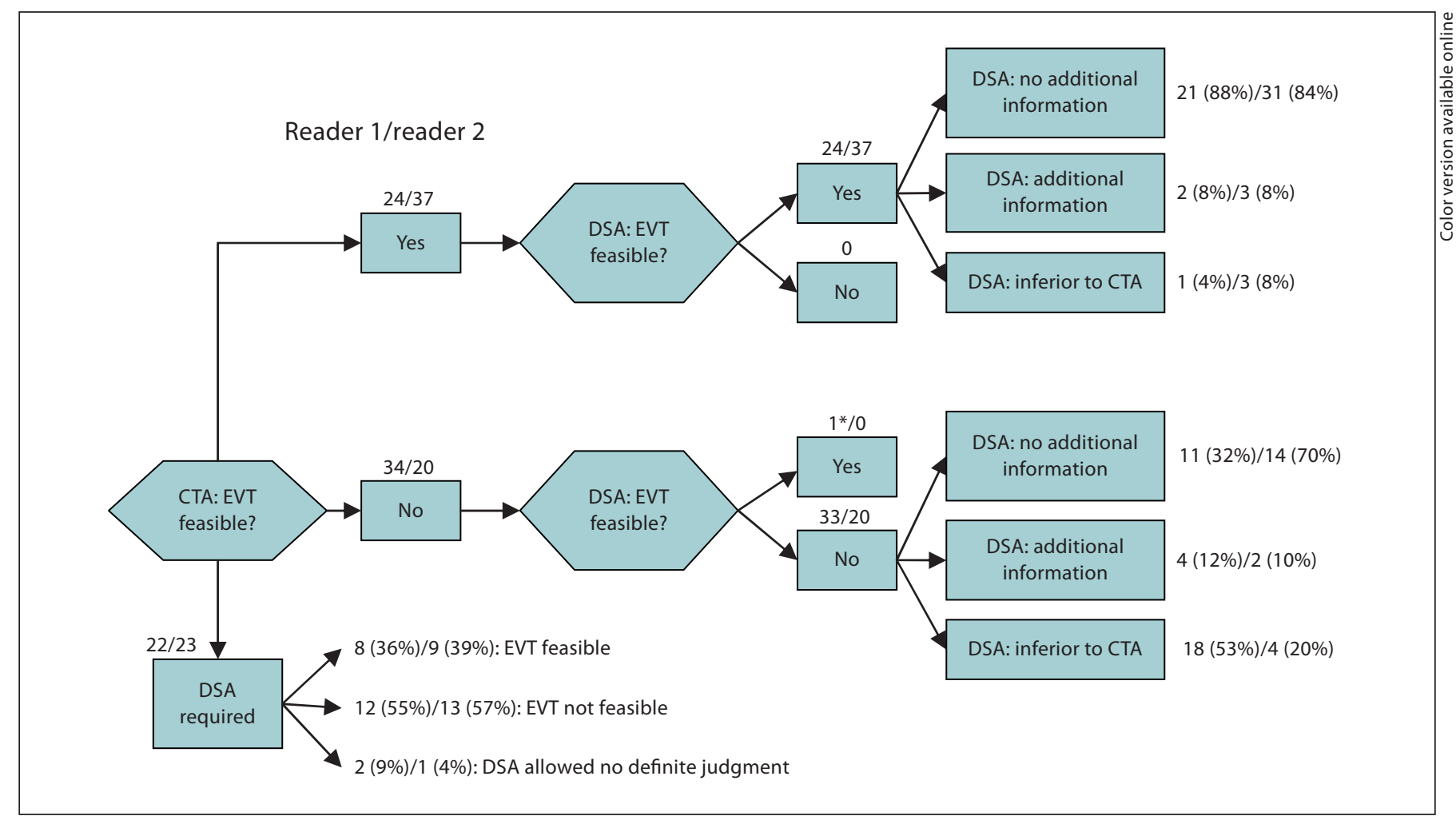

Fig. 1. Flowchart of judgments made by both readers regarding feasibility of EVT of target aneurysm with CTA both before and after viewing DSA. Viewing DSA after CTA changed the judgment of treatment feasibility in 1 patient by reader $1 .{ }^{*}$ Vasospasm at the time of CTA was the reason for the judgment that the feasibility of EVT of the aneurysm could not be assessed. DSA was performed later, when vasospasm had subsided.

\section{Results}

From 111 consecutive patients with SAH who underwent CTA and DSA, 31 were excluded: 2 patients had traumatic SAH, in 8 patients the DSA was not available for analysis, in 1 patient one of the readers doubted the presence of a target aneurysm on CTA, but not on DSA. Twenty patients were excluded because both CTA and DSA did not reveal an aneurysm. This study is based on the remaining 80 patients ( 24 men, 56 women; mean age $\pm \mathrm{SD}$ was $53 \pm 12$ years, range 19-80).

The locations of target aneurysms by vascular territory were: anterior cerebral artery in 35 patients (44\%), 20 had an internal carotid artery (ICA) aneurysm (25\%), 19 had a middle cerebral artery aneurysm (24\%), and $6 \mathrm{had}$ a posterior circulation aneurysm (8\%). There was no disagreement between the readers regarding the locations of the target aneurysms.

Size distribution of the target aneurysms was as follows: 8 (10\%) aneurysms were $\leq 3 \mathrm{~mm}, 16(20 \%)$ were $>3$ and $\leq 5 \mathrm{~mm}, 46(58 \%)$ were $>5$ and $\leq 10 \mathrm{~mm}$, and 10 (13\%) were $>10 \mathrm{~mm}$.

\section{Feasibility Judgments on EVT and Interobserver Agreement}

Both readers were able to make a definite judgment on feasibility of EVT with CTA alone in 58 (73\%) and 57 (71\%) of the patients, respectively (fig. 1; table 1). DSA results never changed definite judgments made with CTA alone, except in 1 patient by reader 1 who underwent DSA later than CTA. In this patient, reader 1 judged EVT not yet feasible with CTA due to the presence of vasospasm, but the DSA that was performed later did not show any vasospasm and the aneurysm was then judged suitable for EVT. Interobserver agreement on judgments on feasibility of EVT (feasible, not feasible or need for DSA) based on CTA was just fair (Cohen's $\kappa=0.35$; table 1 ; fig. 2). Interobserver agreement on judgments based on both CTA and DSA was similar (Cohen's $\kappa=0.39$; table 2). 


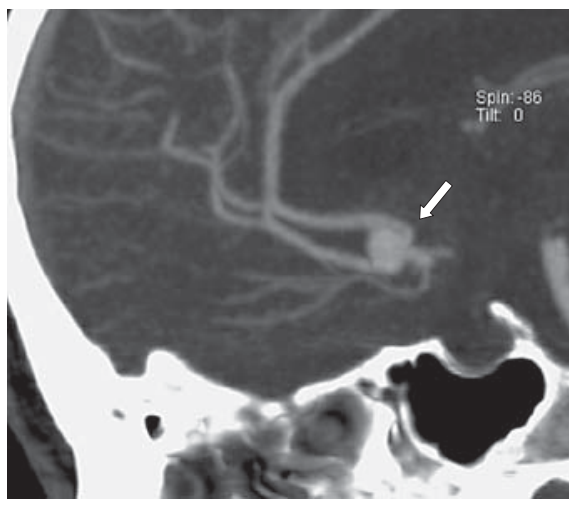

Fig. 2. CTA. In this patient, 1 reader judged EVT as feasible, but the other reader judged it not feasible because of an artery sprouting from the base of the aneurysm (arrow).
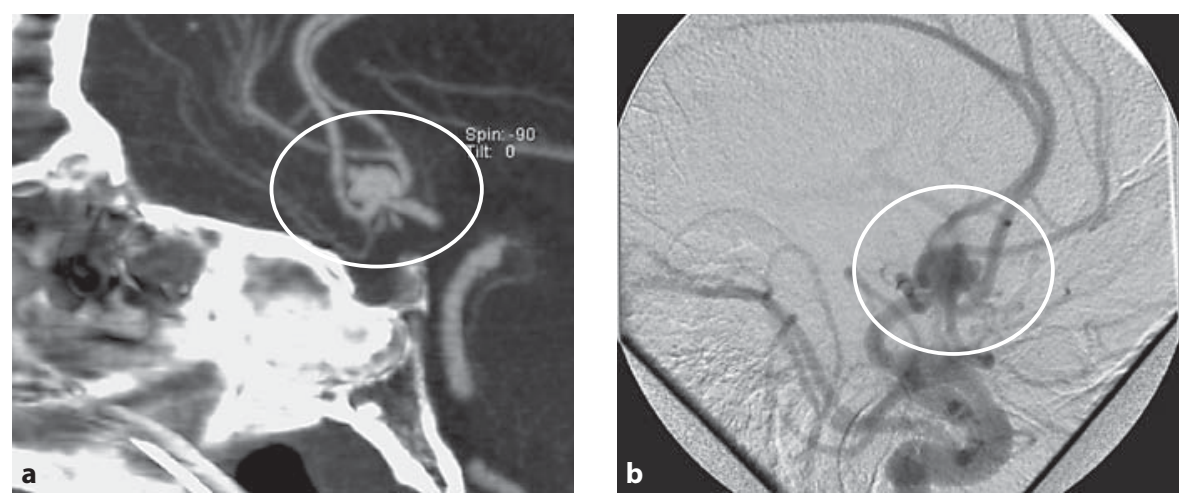

Fig. 3. CTA (a) and DSA (b). In this patient, both readers judged the depiction of anatomical detail of local anatomy around the aneurysm to be visualized more clearly with CTA than with DSA, and therefore judged DSA as inferior to CTA.
Table 1. Interobserver agreement on feasibility of EVT of ruptured aneurysms with CTA alone

\begin{tabular}{lrrrr}
\hline CTA & \multicolumn{3}{l}{ Reader 2: EVT feasible? } & Total \\
\cline { 2 - 4 } & yes & no & DSA needed & \\
\hline Reader 1: EVT feasible? & & & & \\
$\quad$ yes & 21 & 1 & 2 & 24 \\
$\quad$ no & 7 & 15 & 12 & 34 \\
$\quad$ DSA needed & 9 & 4 & 9 & 22 \\
\cline { 2 - 4 } Total & 37 & 20 & 23 & 80 \\
\hline
\end{tabular}

Cohen's $\kappa=0.35$.

We also calculated interobserver agreement with CTA before and after DSA on feasibility judgments (yes vs. no) in the patients in whom both readers made a definite judgment. For the judgments with CTA alone $(n=44), \kappa$ was 0.64 , and with both CTA and DSA, $\kappa$ was $0.42(\mathrm{n}=$ 77). Next, we calculated interobserver agreement $(n=80)$ for definite judgments on feasibility with CTA alone (yes or no) versus the need for DSA (DSA required; fig. 1 ). $\kappa$ was particularly low (0.16).

Comparison of DSA with CTA when EVT Was Judged Feasible with CTA Alone

In this group (24 and 37 patients for reader 1 and 2, respectively), DSA yielded no additional information with regard to the treatment decision of the target aneurysm in 21 and 31 patients.
Table 2. Interobserver agreement on feasibility of EVT of ruptured aneurysms with CTA and DSA thereafter

\begin{tabular}{lrrrr}
\hline CTA and DSA & \multicolumn{3}{c}{ Reader 2: EVT feasible? } & \multirow{2}{*}{ Total } \\
\cline { 2 - 4 } & yes & no & no judgment & \\
\hline Reader 1: EVT feasible? & & & & \\
$\quad$ yes & 28 & 5 & 0 & 33 \\
$\quad$ no & 18 & 26 & 1 & 45 \\
$\quad$ no judgment & 0 & 2 & 0 & 2 \\
\cline { 2 - 4 } Total & 46 & 33 & 1 & 80 \\
\hline
\end{tabular}

Cohen's $\kappa=0.39$.

DSA yielded additional information in 2 patients (reader 1) and 3 patients (reader 2). In these 2 patients (reader 1), the target aneurysm appeared even better accessible for EVT with DSA than with CTA in 1 patient, and in the other patient, the target aneurysm was judged suitable for EVT with balloon remodeling with CTA, whereas with DSA, it was judged suitable for EVT without balloon remodeling. In the other 3 patients (reader 2 ), the anatomical detail of the aneurysm neck or the neckto-dome ratio seen on DSA was judged as complementary to the information by CTA.

DSA was judged as inferior to CTA in 1 of 24 patients by reader 1 and in 3 of 37 patients by reader 2 (fig. 1, 3), because of less detail of local anatomy around the target aneurysm. 
Comparison of DSA with CTA when EVT Was Judged Not Feasible with CTA Alone

In this group (34 and 20 patients for reader 1 and 2, respectively), DSA yielded no additional information with regard to the treatment decision of the target aneurysm in 11 and 14 patients (fig. 1).

DSA yielded additional information in 4 patients (reader 1) and 2 patients (reader 2). In these 4 patients (reader 1), visualization of the aneurysm, the aneurysmal neck and surrounding vessels was judged as superior to CTA $(n=2)$. Kinking of the ICA was seen on DSA in 1 patient and filling of the target (anterior cerebral artery) aneurysm was from the left side (DSA) instead of the right side (with CTA) in another, but vasospasm was more extensive on CTA resulting in a wrong assessment. In the other 2 patients (reader 2), the ICA was additionally seen to be occluded on DSA compared with CTA in 1 patient, and in the other, the anatomical detail of the complex target aneurysm was better seen on DSA.

In 18 (reader 1) and 4 (reader 2) patients, DSAs were judged as inferior to CTA, because of less anatomical detail seen on DSA.

Reasons for Requiring DSA when CTA Was Regarded as Insufficient for a Definite Judgment on EVT

Reader 1 and 2 required DSA for a treatment decision in 22 and 23 patients, respectively. Reasons for requiring DSA were unclear relation of sprouting arteries from the target aneurysm on CTA ( $\mathrm{n}=12$ and 12 , respectively), vessels sprouting from the aneurysm ( $\mathrm{n}=7$ and 2$)$, vasospasm and insufficient contrast filling of the target area ( $\mathrm{n}=1$ and 2$)$, as well as insufficient CTA quality $(\mathrm{n}=1$, reader 1). In 1 patient, a left-sided ICA aneurysm was doubted to be the target aneurysm because the blood distribution on the diagnostic CT strongly suggested a left middle cerebral artery aneurysm according to reader 1 . However, on DSA, the same aneurysm was seen, and the reader concluded it was the ruptured aneurysm.

Reader 2 had other reasons for requiring DSA in the remaining patients: the aneurysm was too small on CTA, the aneurysm neck could not be evaluated well enough, or the neck seemed too wide.

Relation between Size and Location of the Target Aneurysm and Presence of Vasospasm and Feasibility Judgments

Size and location of the target aneurysm and presence of vasospasm did not influence treatment decisions with CTA alone or with CTA and DSA (data not shown). The smallest aneurysm that allowed for a treatment decision by both readers with CTA alone measured $1.3 \mathrm{~mm}$ on CTA. There was 1 smaller aneurysm measuring $1.2 \mathrm{~mm}$ for which both readers required DSA but disagreed on feasibility of EVT of the aneurysm after DSA.

\section{Discussion}

In this series of patients, assessment of the feasibility of EVT of ruptured intracranial aneurysms could be performed reliably with 16-detector row CTA without pretreatment conventional angiography in the majority of patients (about three quarters in our study), even in those with very small aneurysms. The size of the target aneurysm, its location and the presence of vasospasm did not influence treatment decision. When CTA was regarded as sufficient to make a decision on feasibility of EVT, the additional value of DSA after CTA was indeed limited to confirmation of the findings on CTA without affecting the treatment decisions based on CTA alone. In these patients, DSA was even judged as inferior to CTA in 12-33\% of the patients. In about a quarter of the patients, CTA alone provided insufficient information to warrant a definite treatment decision without DSA. Finally, considerable interobserver variability existed in feasibility judgments of EVT, both with or without additional DSA.

Our study has some limitations. First, our results may not be applicable to hospitals where 3-dimensional (3D) DSA is used in the evaluation of intracranial aneurysms. 3D DSA has been shown to be able to provide superior anatomical detail of ruptured aneurysms and surrounding arteries as compared with 2D DSA [10-12]. Thus, comparison of CTA with 3D DSA may have yielded a lower amount of DSAs that were considered inferior to CTA. Second, we did not include patients in whom no aneurysm was found or in whom the readers did not agree on the presence of a target aneurysm. Therefore, our results apply only to patients with a readily identified target aneurysm but not to those with an aneurysm with a size of approximately $1 \mathrm{~mm}$ or less or in whom the presence of an aneurysm is equivocal. Third, we chose a pragmatic design that allowed both readers to judge feasibility of EVT by their own standards to approach daily clinical routine. Therefore, we did not use explicit criteria for feasibility. We acknowledge that this may be an important reason for the significant interobserver variability between the readers and that external validity of our findings may be limited by this method. Fourth, we did not compare the readers' judgments with the patients' actual treatments. We do not consider this a weakness of our 
study, because we have no reason to believe that the judgments made in this study would not have been applicable in a true clinical setting.

Few previous studies exist on evaluation and treatment planning of intracranial aneurysms with 16-detector row CTA and only 1 of these studies specifically addressed the role of 16-detector row CTA for EVT planning [13]. In this other study [13], feasibility of EVT was assessed by means of 16 -detector row CTA in 62 aneurysmal SAH patients. The authors found that suitability for EVT was correctly assessed by CTA in 69 of 74 target aneurysms (93\%, 2 false-positive and 3 false-negative judgments) in these patients, when compared with the reference standard that consisted of the actual treatment performed, i.e. successful placement of coils in the target aneurysm. The authors concluded that multidetector CTA can be used to assess suitability for EVT. In contrast to the aforementioned study, the readers in our study had the possibility to request DSA when CTA was judged insufficient for a definite decision on suitability for EVT. We feel that this design reflects clinical practice more accurately. Further, because DSA was assessed directly after CTA, the additional value of DSA over CTA could be assessed in our study. We found no additional value of DSA after a definite treatment decision with CTA, whereas in the other study [13], DSA yielded the additional information in the 5 patients that had incorrect assessment of suitability for EVT with CTA.

In another study [6], 57 subjects were studied who underwent both 16-detector row CTA and DSA. A vascular neurosurgeon considered CTA alone sufficient for the assessment of suitability for surgical treatment in all but 5 cases in which he required DSA for further anatomical detail. However, the value of CTA for the assessment of suitability for EVT was not studied. We chose not to evaluate the DSA results separately, because in clinical practice, judgments would also include the results of both examinations when performed. With this strategy, we aimed at determining if DSA had an additional value.

Two recent studies reported on the value of 4-detector row CTA in therapeutic management, both surgically and endovascularly, of intracranial aneurysms $[14,15]$. In these studies, DSA was performed only when the CTA was judged to provide insufficient information. Further, EVT was performed in the minority of patients. In the first study, initial suitability of the ruptured aneurysm with CTA for EVT was withdrawn after viewing the DSA in 5 of 26 cases [14]. In the second study, 30 of 88 patients with aneurysmal SAH successfully underwent EVT without pretreatment DSA, but the authors did not mention whether the intraprocedural DSA revealed unexpected findings that complicated the procedure [15]. In 15 of the 16 patients for whom the neurosurgeon requested DSA, the ruptured aneurysm was clipped. The results of this study seem to be in line with our findings, but potential additional information by DSA was not investigated.

Other previous clinical studies on the value of CTA in treatment planning used either 4-detector row or singledetector CT scanners or addressed implementation of CTA in the planning of surgical treatment $[1,3-5,16,17]$. Comparison of our findings with these studies is difficult, because 16-detector row CT scanners have better spatial and temporal resolution $[1,18]$ and our study primarily aimed at assessing the value of CTA in the planning of EVT. Further, the requirements posed on any angiographic investigation may differ for endovascular or surgical therapy, for instance with respect to flow dynamics [19].

Some clinicians still argue for DSA and against CTA as first-line diagnostic investigation in aneurysmal SAH [20] because of the inferior diagnostic accuracy in the detection of aneurysms with CTA. The present paper deals with the diagnostic information needed to take a therapeutical decision once an aneurysm is detected. CTA is increasingly performed as the first-line investigation in patients with SAH. The present paper shows that once an aneurysm is detected, decisions can be made with CTA alone in most of the cases.

In conclusion, our results suggest that a ruptured intracranial aneurysm that is suitable for endovascular occlusion as assessed by 16-detector row CTA by an interventional neuroradiologist could be safely scheduled for endovascular occlusion immediately, without pretreatment DSA, in about three quarters of the patients. This strategy will improve the management flow of patients with ruptured intracranial aneurysms. However, interobserver variability in the assessment of feasibility of EVT of ruptured aneurysms was considerable.

\section{Acknowledgements}

The authors thank Wibeke van Leeuwen and Caroline van Bavel of the Radiology Trial Office of the Erasmus MC for their excellent secretarial help. 


\section{References}

1 Goddard AJP, Tan G, Becker J: Computed tomography angiography for the detection and characterization of intra-cranial aneurysms: current status. Clin Radiol 2005;60:12211236.

$\checkmark 2$ Chawla S: Advances in multidetector computed tomography. Applications in neuroradiology. J Comput Assist Tomogr 2004;28: S12-S16.

-3 Wintermark M, Uske A, Chalaron M, Regli L, Maeder P, Meuli R, Schnyder P, Binaghi S: Multislice computerized tomography angiography in the evaluation of intracranial aneurysms: a comparison with intraarterial digital subtraction angiography. J Neurosurg 2003;98:828-836.

4 Kangasniemi M, Mäkelä T, Koskinen S, Porras M, Poussa K, Hernesniemi J: Detection of intracranial aneurysms with two-dimensional and three-dimensional multislice helical computed tomographic angiography. Neurosurgery 2004;54:336-341.

5 Dammert S, Krings T, Moller-Hartmann W, Ueffing E, Hans FJ, Willmes K, Mull M, Thron A: Detection of intracranial aneurysms with multislice CT: comparison with conventional angiography. Neuroradiology 2004;46:427-434.

-6 Tipper G, U-King-Im JM, Price SJ, Trivedi RA, Cross JJ, Higgins NJ, Farmer R, Wat J, Kirollos R, Kirkpatrick PJ, Antoun NM, Gillard JH: Detection and evaluation of intracranial aneurysms with 16-row multislice CT angiography. Clin Radiol 2005;60:565572.
7 Molyneux A, Kerr R, Stratton I, Sandercock P, Clarke M, Shrimpton J, Holman R; International Subarachnoid Aneurysm Trial (ISAT) Collaborative Group: International Subarachnoid Aneurysm Trial (ISAT) of neurosurgical clipping versus endovascular coiling in 2143 patients with ruptured intracranial aneurysms: a randomised trial. Lancet 2002;360:1267-1274.

8 Yasargil MG: Microneurosurgery. Stuttgart, Georg Thieme, 1984, vol 1.

9 Landis JR, Koch GG: The measurement of observer agreement for categorical data. Biometrics 1977;33:159-174.

10 Missler U, Hundt C, Wiesmann M, Mayer T, Brückmann H: Three-dimensional reconstructed rotational digital subtraction angiography in planning treatment of intracranial aneurysms. Eur Radiol 2000;10:564568.

-11 Hochmuth A, Spetzger U, Schumacher M: Comparison of three-dimensional rotational angiography with digital subtraction angiography in the assessment of ruptured cerebral aneurysms. Am J Neuroradiol 2002;23: 1199-1205.

12 Albuquerque FC, Spetzler RF, Zabramski JM, McDougall CG: Effects of three-dimensional angiography on the coiling of cerebral aneurysms. Neurosurgery 2002;51:597-606.

13 Papke K, Kuhl CK, Fruth M, Haupt C, Schlunz-Hendann M, Sauner D, Fiebich M, Bani A, Brassel F: Intracranial aneurysms: role of multidetector CT angiography in diagnosis and endovascular therapy planning. Radiology 2007;244:532-540.

-14 Dehdashti AR, Rufenacht DA, Delavelle J, Reverdin A, De Tribolet N: Therapeutic decision and management of aneurysmal subarachnoid haemorrhage based on computed tomographic angiography. Br J Neurosurg 2003;17:46-53.
15 Hoh BL, Cheung AC, Rabinov JD, Pryor JC, Carter BS, Ogilvy CS: Results of a prospective protocol of computed tomographic angiography in place of catheter angiography as the only diagnostic and pretreatment planning study for cerebral aneurysms by a combined neurovascular team. Neurosurgery 2004;54:1329-1342.

16 Velthuis BK, Van Leeuwen MS, Witkamp TD, Ramos LMP, Berkelbach van der Sprenkel JW, Rinkel GJE: Computerized tomography angiography in patients with subarachnoid hemorrhage: from aneurysm detection to treatment without conventional angiography. J Neurosurg 1999;91:761-767.

17 Boet R, Poon WS, Lam JMK, Yu SCH: The surgical treatment of intracranial aneurysms based on computer tomographic angiography alone - streamlining the acute management of symptomatic aneurysms. Acta Neurochir 2003;145:101-105

18 Cademartiri F, Luccichenti GL, Van der Lugt A, Pavone P, Pattynama PM, de Feyter PJ, Krestin GP: Sixteen-row multislice computed tomography: basic concepts, protocols, and enhanced clinical applications. Semin Ultrasound CT MR 2004;25:2-16.

19 Velthuis BK, Rinkel GJ, Ramos LM, Witkamp TD, Berkelbach van der Sprenkel JW, Vandertop WP, Van Leeuwen MS: Subarachnoid haemorrhage: aneurysm detection and preoperative evaluation with CT angiography. Radiology 1998;208:423-430.

20 Kallmes DF, Layton K, Marx WF, Tong F: Death by nondiagnosis: why emergent CT angiography should not be done for patients with subarachnoid hemorrhage. Am J Neuroradiol 2007;28:1837-1838. 